La cumulativité des savoirs en sciences sociales

\title{
Cumulativité des connaissances
}

\section{Denise Pumain}

\section{OpenEdition \\ Journals}

Édition électronique

URL : http://journals.openedition.org/ress/357

DOI : $10.4000 /$ ress.357

ISSN : 1663-4446

\section{Éditeur}

Librairie Droz

\section{Édition imprimée}

Date de publication : 1 février 2005

Pagination : $5-12$

ISBN : 2-600-00958-2

ISSN : 0048-8046

Référence électronique

Denise Pumain, «Cumulativité des connaissances », Revue européenne des sciences sociales [En ligne], XLIII-131 | 2005, mis en ligne le 04 novembre 2009, consulté le 20 avril 2019. URL : http:// journals.openedition.org/ress/357 ; DOI : 10.4000/ress.357 


\section{Denise PUMAIN}

\section{CUMULATIVITÉ DES CONNAISSANCES}

Comment les savoirs produits par les sciences sociales peuvent-ils s'accumuler, se cumuler, au cours du temps dans une discipline, et entre les différentes disciplines? La question est difficile en raison des multiples facettes impliquées par ces connaissances (théories, concepts, données statistiques ou faits empiriques), mais aussi de conditions épistémologiques particulières. La cumulativité des connaissances en sciences sociales fait débat: la multiplicité des concepts et des théories, la fluidité des définitions et la diversité de leurs interprétations, laissent sceptiques de nombreux chercheurs, pas nécessairement relativistes, qui voient dans la cumulativité tout au plus un horizon d'attente, probablement irréalisable. En effet, l'idée que les savoirs peuvent s'accumuler se situe en aval de croyances très controversées, comme celle du progrès dans les sciences sociales, elle suppose aussi peut-être une forme de réalisme dans la construction du sens. La notion traduite semble poser moins de problèmes aux épistémologues anglais ou américains, pour qui la knowledge accumulation semblerait aller bien plus de soi (par ex., Mahoney, Ruescheweyer, 2003, National Academies, 2003), même si elle suscite parfois une dénonciation morale, aussi vigoureuse que celle de l'accumulation du capital! Il est vrai que les enjeux de la cumulativité des savoirs dans les sociétés modernes ont déjà entraîné l'apparition d'une discipline nouvelle, «l'économie des connaissances », déjà très riche de manuels et formations spécialisées... Bien évidemment, ce processus d'accumulation ne saurait consister en une simple addition, puisque même dans le cas des enquêtes et des inventaires statistiques, il implique des formes de recomposition et de révision qui amènent à s'interroger sur les modalités de construction de ces savoirs, dans la durée. Comment s'effectue pratiquement le travail d'articulation des théories et des concepts anciens et nouveaux dans nos disciplines? La question peut s'inscrire dans l'examen de trois sous-ensembles de conditions, sociologiques, technologiques et épistémologiques, qui sont évidemment étroitement liées mais que l'on peut dissocier pour l'analyse. Elle pose aussi le problème plus général de la transférabilité des concepts.

\section{CUMULATIVITÉ ET INSTITUTIONS SCIENTIFIQUES}

Dans une perspective d'histoire ou de sociologie des sciences, la question des savoirs cumulables ou accumulés, peut susciter des investigations dans au moins trois grandes directions: 
1. Quelles sont les conditions sociales de l'acceptation d'une théorie nouvelle, de sa reconnaissance comme invention ou innovation, non pas en tant que telle, dans le sens où nous l'avons déjà traité (Bridel, 2004), mais en termes de son intégration à des corpus existants? Selon quelles pratiques se construisent les «chaînes de traduction» qui assurent la circulation des concepts nouveaux? Quels réseaux sociaux sont utilisés pour relayer l'insertion des nouvelles ontologies, et, plus rarement, celles de nouveaux schèmes interprétatifs ou paradigmes?

2. La cumulativité des connaissances est très souvent prônée par les institutions de gestion et de coordination de la recherche, même s'il est bien souvent reconnu que la mise en œuvre en est assez difficile. Comment organise-t-on la production de connaissances nouvelles, quelles incitations, quelles procédures en favorisent l'émergence, et l'intégration à des savoirs plus anciens? Par quels moyens s'assure-t-on de la mise en relation et de la conservation des savoirs acquis? Il s'agit en somme d'analyser comment s'établissent les rapports entre anciens et nouveaux programmes de recherche. Il importe aussi de comprendre comment la cumulativité est prise en compte par les instances d'évaluation de la recherche.

3. La plupart des institutions d'enseignement, tout comme l'UNESCO qui y a consacré un colloque récent (2003), mettent en avant la cumulativité des savoirs enseignés. Quelles sont les modalités de transmission des connaissances acquises, sous quelle forme, personnelle ou collective, globale ou fragmentée, directe ou médiatisée? Quels filtrages et quelles révisions interviennent lors de ces passations?

\section{CONDITIONS TECHNIQUES}

Une partie de l'activité scientifique se résout dans la production d'informations, archivables et donc cumulables: résultats d'enquêtes, dénombrements, inventaires, typologies, jurisprudences, archives... La conception traditionnelle du musée, tout comme l'institutionnalisation plus récente des «Data Archives» en sciences sociales, se fondent d'abord sur l'idée de cumulativité. Certes, le caractère cumulable des informations dépend très largement de la façon dont ces données ont été construites, des théories et des procédures d'observation qui ont présidé à leur élaboration, comme le montre bien l'histoire des statistiques (Desrozières, 1993). Mais il ne faut pas oublier que les modalités du stockage et de la gestion des sources d'information sont essentielles aux possibilités de réutilisation et donc d'accumulation de ces savoirs. Comment les bases de données sont-elles conçues, sur quels supports et pour quelle diffusion, avec quelle précision les méta-données sont-elles renseignées? Comment s'opère la révision des nomenclatures? Quelles sont les conditions de l'obsolescence des données ou de la sélection des informations au cours du temps?

Les bouleversements récents des moyens techniques de gestion et de stockage des informations ont introduit d'importantes modifications, tant en termes des conditions d'accès à l'information que des transformations induites du contenu 
des informations archivées. La cumulativité des connaissances s'en trouve-t-elle renforcée, ou faut-il constater d'irréparables «pertes en ligne» des connaissances non intégrées dans les nouveaux systèmes? Il s'agit ici d'interroger les médiations entre les archives, les données, et la production des savoirs scientifiques. En ouvrant le comparatisme aux dimensions du monde entier, en favorisant les circulations d'informations entre des domaines disciplinaires ou institutionnels jusqu'ici cloisonnés, les usages croissants d'Internet pour l'accès aux connaissances vont modifier profondément les conditions de cumulativité des savoirs scientifiques. La structuration et la circulation des connaissances sont bouleversées par les méthodes d'indexation et de recherche en langage naturel, par l'organisation des liens en hypertexte, plus généralement par les techniques du multimédia. Que l'on applaudisse à la possible immersion de tous dans un bain de savoir universel et aux forme démocratisées de la participation à la connaissance, que l'on s'inquiète de l'appauvrissement sémantique et de la déconstruction des savoirs, ou que l'on dénonce la pseudo-démocratisation d'un système qui recrée dans les faits d'énormes inégalités dans la hiérarchie de l'accès à l'information et à sa maîtrise, on ne peut éviter de considérer l'hypothèse selon laquelle les effets de cette révolution technologique, encore peu discernables, soient d'une magnitude supérieure à ceux qu'a suscités l'invention de l'imprimerie, depuis le XVI siècle.

\section{CONTEXTES ÉPISTÉMOLOGIQUES}

Qu'est-ce qui conditionne la révision des connaissances scientifiques, ou plus généralement des «croyances réflexives »? Sans revenir sur les interrogations liées à l'administration de la preuve, bien étudiées à Arolla en 2003, la notion de cumulativité des savoirs est fortement liée au statut des connaissances nouvelles et aux conditions de remplacement d'une théorie par une autre. Parmi les hypothèses émises quant aux modalités du changement théorique, quelles sont celles qui conviendraient aux sciences sociales: sont-elles soumises à un régime de discontinuité (une théorie chasse l'autre) la discontinuité étant liée à l'incommensurabilité des théories, ancienne et nouvelle, comme le supposent la révolution kuhnienne à l'occasion du changement de paradigme, ou encore le contextualisme de la philosophie foucaldienne? Peut-on imaginer un autre processus, plus continuiste, qui permettrait la réinterprétation d'une théorie ancienne dans une nouvelle, plus englobante, ou une évolution contrôlée du contenu des concepts? La cumulativité des connaissances pourrait alors se produire à partir des interférences entre des courants théoriques distincts à l'origine, sous forme d'hybridations entre théories différentes ou de réhabilitation d'hypothèses abandonnées. Le constat dominant reste celui de la persistance d'une pluralité de théories concurrentes, qu'elles se présentent comme contradictoires ou simplement alternatives. Mais qu'en est-il de leur évolution? Entre communication et conservation du savoir, observe-t-on une sélection entre les théories, selon l'hypothèse darwinienne de survie de la plus apte, ou selon un processus évolutionniste de lock-in, éventuellement non optimal' ${ }^{1}$ ?

Un exemple bien connu est la persistance des représentations proposées par Zipf pour les distributions très inégales, qui inversent le formalisme statistique habituel. 
Quelle est la place de la mise à l'épreuve par l'observation empirique dans ces processus? En particulier, comment se joue le couple formalisation-observation dans les disciplines qui ont développé un corpus formel important, comme l'économie? Ces questions peuvent se poser, non seulement à l'intérieur du débat scientifique, mais dans le contexte des interactions entre science et société. A moyen terme, certains enjeux politiques semblent peser lourdement sur les vulgates des sciences sociales, comme le suggèrent les débats récents autour du rôle joué par les théories économiques valorisées par le FMI dans le devenir des pays pauvres, ou encore des effets auto-réalisateurs (et dévastateurs d'entreprises) des théories à la mode dans les écoles de gestion - sans parler des toujours controversées «leçons de l'histoire »!

En préalable, la question de la spécificité des «sciences historiques» par rapport aux sciences dites «dures » ou «exactes» est posée. La condition indiquée par J.C. Passeron, en citant Merton (« la sociologie n'a pas et ne peut prendre la forme d'un savoir cumulatif, c'est-à-dire d'un savoir dont un paradigme théorique organiserait les connaissances cumulées », page 364) n'est-elle pas excessivement exigeante? Les connaissances des sciences dites «dures», expérimentales, sontelles vraiment organisées en pratique dans un même paradigme théorique? Beaucoup des énoncés de ces disciplines s'inscrivent en pratique dans des constructions provisoires, ou mal intégrées, voire inexistantes (par exemple, on ne sait pas expliquer pourquoi l'eau est liquide). La multiplicité des contextes différents, dans l'espace et dans le temps, est aussi invoquée par J.C. Passeron comme un obstacle rédhibitoire à la comparaison des cas et donc à la cumulativité des connaissances. Cependant, puisque l'histoire du monde social est «finie», le savoir acquis sur cette énumération de cas doit en principe être cumulable, même s'il s'agit d'interprétations partielles, voire erronées, toujours révisables. L'idée selon laquelle seules les sciences expérimentales seraient capables d'une énonciation complète des «traits pertinents du contexte pour la validité de la mesure ou de l'observation considérée » (p. 368) semble refléter une ambition de connaissance excessive, qui crédite les sciences «dures» bien au-delà de ce qu'elles sont: les notions de marge d'erreur liée au contexte de l'expérience et de niveau de précision des mesures sont en fait toujours admises et prises en considération par les disciplines expérimentales. Ces conditions préalables à la cumulativité des connaissances n'introduiraient selon nous qu'une différence de degré et pas de nature entre les sciences de la nature, qui ne sont d'ailleurs pas toutes expérimentales, et les sciences sociales.

En effet, le contexte contemporain d'une réflexion commune proposée à l'ensemble des sciences sur le paradigme de la complexité, invite à réviser le constat banal de la complexité des constructions sociales, appelé à la rescousse pour leur dénier tout droit à la cumulativité des connaissances et plus largement aux normes classiques de la scientificité. Les théories de la complexité se posent volontiers comme une nouvelle forme de rationalité, qui effacerait la différence entre les épistémologies nomothétique et idiographique. Toute l'activité scientifique s'occuperait d'objets uniques. Si le modèle newtonien de la science est abandonné par les sciences dures, si le principe nomothétique n'est plus soutenable et si tout objet devient idiographique, même dans les sciences physiques, si la question de l'échelle de précision des assertions et de leur contexte de validité arrive au centre des investigations (par exemple entre physique quantique et physique de notre 
monde sensible), si les notions d'ordre et de rationalité admettent une part de subjectivité, si l'imprévisibilité prend la place du déterminisme, et si tous les processus admettent une flèche du temps irréversible (Prigogine, 2001), alors, c'est l'épistémologie des sciences sociales qui devient la référence pour toutes les sciences, et qui est placée au cœur de la réflexion sur le savoir. Parmi d'autres, la question de la cumulativité se pose sous un jour nouveau, entre d'une part les tentations réductrices d'une «physique sociale» désormais revendiquée par des physiciens au nom de l'universalité des «sciences de la complexité», mais prompte à sélectionner dans l'univers des observations possibles du monde social celles qui sont le plus aptes à conforter l'habitus méthodologique de la répétabilité des configurations et des processus (voir par exemple les productions de l'«éconophysique», Durlauf, 2003), et d'autre part les extravagances multiformes des tenants des postures postmodernes récusant tout asservissement aux objectifs et aux protocoles scientifiques. Dans ce nouveau contexte épistémologique, l'un des enjeux d'une meilleure considération de la cumulativité des connaissances par les sciences sociales devient la valorisation des résultats de leur activité, face aux menaces d'exclusion par ces deux courants pourtant antagonistes.

\section{CUMULATIVITÉ ET TRANSFERT DE CONCEPTS ENTRE DISCIPLINES}

«Par cumulativité on entend classiquement la possibilité d'intégrer les résultats d'un grand nombre d'observations et d'expériences dans l'unité d'un modèle susceptible de les déduire» (Berthelot, 1996, p. 74). Parmi les principaux obstacles à cette cumulativité en sciences sociales, Michel Berthelot, à la suite de Jean-Claude Passeron et Raymond Boudon, relève «l'absence d'un langage [comme les mathématiques] susceptible d'intégrer ces diverses dimensions » et «la concurrence des langages existants pour imposer leur point de vue» (p. 88). Il y ajoute «le caractère inépuisable des tableaux empiriques», mais cet argument paraît de plus en plus contestable, du fait de l'évolution des faits envisagés par les sciences physiques, et bien sûr de celles du vivant, conduites à particulariser les objets qu'elles étudient.

Se donner le projet d'une accumulation des savoirs des sciences sociales implique de se demander jusqu'à quel point les théories et les concepts des sciences sociales seraient si étroitement liés au contexte qui les a vus émerger que l'évolution de celui-ci les condamnerait à l'obsolescence et rendrait vaine toute recherche de cumulativité. Les tentatives de révision progressive et d'adaptation des théories et des concepts, d'enrichissement des contenus notionnels, sont-elles contradictoires avec le respect des conditions de validité qui prévalaient lorsqu'ils ont été produits? Les langages élaborés par des théories concurrentes sont-ils aussi complètement hermétiques les uns aux autres qu'ils le prétendent? Plus largement les hypothèses discontinuistes, de l'incommensurabilité des connaissances produites dans des contextes paradigmatiques différents, chez Thomas Kuhn comme chez Michel Foucault, invalideraient-elles tout projet de cumulativité des savoirs scientifiques? La posture postmoderne énumère à l'envi des justifications à ce doute absolu. Si l'on ne souscrit pas à ces hypothèses, elles invitent 
cependant à considérer avec toute la précaution nécessaire le processus de transfert des connaissances, non seulement au cours du temps dans une discipline, mais aussi entre les disciplines, qui se sont construites plus ou moins arbitrairement sur certains aspects et certaines échelles de nos représentations du monde.

En effet, nos disciplines se sont construites plus ou moins arbitrairement sur certains aspects et certaines échelles de nos représentations du monde. Le paradigme contemporain de la complexité, qui met l'accent sur les effets d'interaction entre les échelles, pour comprendre l'émergence des structures, des formes, des institutions..., invite à repenser ces catégories, et souligne la nécessité de développer des interfaces entre les disciplines des sciences sociales. Si l'on réussit à faire communiquer les langages disciplinaires entre eux, on aura levé l'un des obstacles essentiels à la cumulativité des connaissances, qui résiderait dans la diversité de leurs approches théoriques, l'incommunicabilité de leurs langages. Encore faut-il que cette opération construise un savoir nouveau, sans se limiter à une simple reformulation, et il convient de déterminer dans quelle mesure la transcription des savoirs anciens dans un nouveau paradigme peut contribuer à l'accumulation des connaissances.

Le groupe «raison et rationalités » s'est donné depuis longtemps les moyens de naviguer entre les deux écueils évoqués à propos de la complexité, celui du réductionnisme et celui du relativisme. Mais à quelles conditions son expérience de l'échange interdisciplinaire peut-elle produire des connaissances cumulables? Dès lors qu'il s'agit, non pas d'additionner des connaissances, mais de produire des savoirs articulant explicitement des concepts issus de disciplines différentes, la question centrale devient celle de la transférabilité des concepts entre les disciplines, ce qui n'est pas tout à fait l'analogue de leur évolution au cours du temps dans une même discipline.

Deux méthodes paraissent susceptibles de permettre cette élaboration. Dans les deux cas, il s'agit de déconstruire des approches disciplinaires en vue de reconstruire un autre savoir à un niveau plus englobant. La première méthode consisterait à partir d'un savoir disciplinaire, enraciné dans un point de vue particulier, mais à le mettre en représentation dans un contexte plus large, mobilisant éventuellement des interrogations de sens commun (à partir d'une «bonne histoire» par exemple) et restituant l'argumentaire et la force de conviction de l'enquête scientifique. En somme, il s'agit de faire parler chacun sur ce qu'il aurait envie de communiquer aux autres, en tant que résultat ou découverte fondamental de sa discipline, qui réponde à une question d'importance générale. Ces acquis marqués par une approche disciplinaire peuvent présenter une valeur générale pour l'ensemble des sciences sociales, et seraient alors inscrits dans l'état provisoire des connaissances cumulables. Il se peut aussi que leur communication révèle qu'il doivent être complétés par des aspects élaborés dans d'autres disciplines, ou rapprochés d'autres notions, et ce sont ces articulations qui formeraient le nouveau contexte dans lequel ce concept est présenté.

La seconde méthode consisterait au contraire à se placer aux marges des disciplines et à y rechercher des concepts partageables par d'autres sciences sociales, concepts «exportables », ou «négociables », qu'il s'agit d'enrichir par le croisement interdisciplinaire. Cette deuxième approche est plus difficile que la première. Elle a donné lieu à des expériences de travail multidisciplinaire sur un objet commun, notamment grâce à la recherche incitative, et semble avoir apporté 
parfois de bons résultats, par exemple dans le domaine des sciences cognitives, mais elle paraît avoir échoué à produire un savoir commun des sciences sociales à propos de la ville. Faut-il songer alors, non pas à mettre des savoirs dans un jeu complémentaire sur un même objet, mais à rapprocher plus en amont des points de vue et des méthodes communs, en somme inventer l'équivalent de la biologie moléculaire ou de la chimie physique? A quand une véritable socio-économie, une géohistoire, une vraie économie géographique, une psychologie politique, une science de la cognition sociale? L'exercice est difficile, parce que la reconnaissance institutionnelle des domaines émergents ne va pas de soi, et aussi parce que les sciences mieux reconnues ont tendance à imaginer qu'elles peuvent se passer des acquis des autres. Ainsi N. Durlauf (2003) dénonce-t-il à juste titre les oublis de «l'éconophysique» vis-à-vis des théories de la science économique. Me sera-t-il permis de déplorer que des économistes de l'auto-proclamée «économie géographique»(Fujita, Krugman et Venables, 2001) défendent les théories sociales absurdement rudimentaires d'un Henderson pour expliquer les spécialisations des villes (qui sortiraient toutes armées du choix délibéré d'un seul acteur urbain bien informé) ou nomment en suivrant Cronon des avantages d'agglomération de «première nature» (caractéristiques d'un site non transformées par l'homme) et de «seconde nature» (caractéristiques d'un site aménagé), là où les géographes ont depuis longtemps démontré l'inanité de cette distinction, puisque la valorisation d'un site change avec le temps, et mis au contraire l'accent sur l'intérêt qu'il y a à distinguer selon deux niveaux d'échelle avantages de site et de situation?

On voit bien comment une véritable articulation de concepts peut favoriser une intégration des processus observés à des échelles de temps et d'espace différents, et permettre que des aspects du social jusqu'ici approfondis séparément retrouvent leur cohérence dans un jeu d'interactions. Le propos n'est donc pas de produire un savoir qui serait simplement transverse aux disciplines scientifiques, qui n'en retiendrait que les propriétés communes, comme celles qui sont prises en compte par exemple par les physiciens, dans le projet d'une «nouvelle» science de la complexité, selon ce qui constitue au fond un travail réducteur par rapport au projet des sciences sociales (en ce qu'il s'efforce d'atteindre ce qui constitue les aspects les plus généraux, donc les plus banals, de la complexité sociale, mais avec des formalismes nouveaux, notamment dans les modèles de simulation). Nous pourrions ainsi, tout en produisant des formalismes nouveaux, illustrer la question de la complexité d'une façon bien plus éclairante que les définitions qu'en ont donné les mathématiciens, que ce soit en termes de quantité d'information (Ashby), ou en termes de «surprise» ou d'émergence (Casti). La complexité d'une notion serait mesurée par la diversité des regards disciplinaires nécessaires à son élaboration, à l'intelligibilité des objets ou des processus étudiés, selon un objectif donné de précision des énoncés et des contextes. Ne nous cachons pas la difficulté de l'entreprise, puisqu'il faudra bien, dans ce processus de recomposition, expliciter des pondérations qui pourraient n'exprimer que les rapports de force entre les positions hiérarchisées des différentes disciplines dans la société.

A l'opposé des tentations postmodernes d'éclatement par la distinction poussée à l'extrême, la menace de réduction épistémologique par les sciences «dures » et leur puissance sociale d'éviction (appuyée sur l'efficacité des modèles d'ingénieur) réussiront peut-être à persuader les sciences sociales de mieux 
valoriser et faire connaître les bases cumulatives de leurs savoirs, selon des modalités qu'elles auraient choisies et construites ensemble.

Ne pourrait-on voir là l'heureuse issue d'un apparent paradoxe: partager pour cumuler?

\section{Université Paris I Panthéon-Sorbonne}

\section{RÉFÉRENCES}

Berthelot J.M. 2001, Epistémologie des sciences sociales. Paris, P.U.F.

Berthelot J.M. 1996, Cumulativité et normativité, in Ramagnino N. et Houle G. Sociologie et normativité scientifique. Toulouse, PUM, 73-90.

Bridel P. (ed.) 2004, L'innovation dans les sciences sociales. Genève, Labor et Fides.

Desrozières A. 1993, La politique des grands nombres. Histoire de la raison statistique. Paris, La Découverte.

Durlauf S.N. 2003, Complexity and empirical economics. Santa Fe Institute, Working paper.

Fujita M. Krugman P. Venables A. 1999, The spatial economy: cities, regions and the international trade. Cambridge, MIT Press.

Mahoney J. Ruescheweyer (eds.) 2003, Comparative history analysis in the social sciences. Cambridge.

National Academies, 2003, Workshop on understanding and promoting knowledge accumulation in education.

Passeron J.C. 1991, Le raisonnement sociologique. L'espace non-poppérien du raisonnement naturel. Paris, Nathan.

Prigogine I. 2001, La fin des certitudes. Paris, Odile Jacob (1 $1^{\text {ère }}$ édition 1996). 